\title{
Lactobacillus rhamnosus GG and its SpaC pilus adhesin modulate inflammatory responsiveness and TLR-related gene expression in the fetal human gut
}

\author{
Kriston Ganguli', Maria C. Collado ${ }^{2,3}$, Jaana Rautava ${ }^{4}$, Lei Lu', Reetta Satokari ${ }^{5}$, Ingemar von Ossowski ${ }^{5}$, Justus Reunanen ${ }^{5}$, \\ Willem M. de Vos ${ }^{5}$, Airi Palva ${ }^{5}$, Erika Isolauri, Seppo Salminen², W. Allan Walker ${ }^{1}$ and Samuli Rautava ${ }^{6}$
}

BACKGROUND: Bacterial contact in utero modulates fetal and neonatal immune responses. Maternal probiotic supplementation reduces the risk of immune-mediated disease in the infant. We investigated the immunomodulatory properties of live Lactobacillus rhamnosus GG and its SpaC pilus adhesin in human fetal intestinal models.

METHODS: Tumor necrosis factor (TNF)- $\alpha$ mRNA expression was measured by qPCR in a human fetal intestinal organ culture model exposed to live L. rhamnosus GG and proinflammatory stimuli. Binding of recombinant SpaC pilus protein to intestinal epithelial cells (IECS) was assessed in human fetal intestinal organ culture and the human fetal intestinal epithelial cell line $\mathrm{H} 4$ by immunohistochemistry and immunofluorescence, respectively. TLR-related gene expression in fetal ileal organ culture after exposure to recombinant SpaC was assessed by qPCR.

RESULTS: Live L. rhamnosus GG significantly attenuates pathogen-induced TNF- $\alpha$ mRNA expression in the human fetal gut. Recombinant SpaC protein was found to adhere to the fetal gut and to modulate varying levels of TLR-related gene expression.

CONCLUSION: The human fetal gut is responsive to luminal microbes. L. rhamnosus GG significantly attenuates fetal intestinal inflammatory responses to pathogenic bacteria. The L. rhamnosus GG pilus adhesin SpaC binds to immature human IECs and directly modulates IEC innate immune gene expression.

C ontrary to the prevailing dogma, recent advances in understanding the micro-ecology of the human placenta and fetus suggest that the fetus does not develop devoid of microbial contact (1). Thus far, most research efforts to understand fetal bacterial contact have focused on chorionamnionitis, a major cause of preterm birth and neonatal infection $(1,2)$. Interestingly, however, microbes and microbial DNA can also be regularly detected in amniotic fluid and placentae from healthy term pregnancies (3-5). The presence of microbial DNA in infant meconium samples also suggests a prenatal origin for bacteria (6). The significance of this fetal microbial contact unrelated to infection remains poorly understood.

It has gradually become evident that in utero microbial contact can modulate fetal immune function and this might have an impact on both short- and long-term disease risk (1). Bacteria detected in the placenta after preterm birth are reportedly associated with changes in systemic neonatal inflammatory responses (7). We have recently reported that DNA from nonpathogenic bacteria in the placenta or amniotic fluid can modulate toll-like receptor (TLR)-related gene expression in the fetal gut in term pregnancies without evidence of chorionamnionitis (5). We have also provided data suggesting that fetal intestinal innate immune gene expression can be modulated by maternal supplementation with specific probiotic bacteria (5). This may represent one of the mechanisms by which maternal probiotic supplementation during pregnancy is able to reduce the risk of immune-mediated disease such as eczema in the infant $(8,9)$. To date, the mechanisms responsible for in utero probiotic action have not been characterized.

We hypothesized that Lactobacillus rhamnosus GG (ATCC 53013) (L. rhamnosus GG), one of the most extensively investigated probiotics, might elicit direct immunomodulatory effects in the fetal gut. Moreover, we suggest that pilus structures on the surface of L. rhamnosus GG may be pivotal in this regard. We have recently reported that the adhesion of L. rhamnosus GG is mediated by SpaCBA pili and that the SpaC pilus adhesin, which is positioned at the pilus tip as well as decorates the shaft, is the major binding factor to human intestinal mucus, collagen and intestinal epithelial cells (IECs) (10-13). SpaC has also been shown to stimulate biofilm development and elicit immunomodulatory effects $(14,15)$. We sought to test our hypothesis and investigate the immunomodulatory properties

\footnotetext{
'Mucosal Immunology and Biology Research Center, Massachusetts General Hospital for Children, Boston, Massachusetts; ${ }^{2}$ Functional Foods Forum, University of Turku, Turku, Finland; ${ }^{3}$ Department of Biotechnology, Institute of Agrochemistry and Food Technology, Spanish National Research Council (IATA-CSIC), Valencia, Spain; ${ }^{4}$ nstitute of Dentistry, University of Turku, Turku, Finland; ${ }^{5}$ Department of Veterinary Biosciences, University of Helsinki, Helsinki, Finland; ${ }^{6}$ Department of Paediatrics, University of Turku, Turku, Finland. Correspondence: Samuli Rautava (samrau@utu.fi)
} 
of L. rhamnosus GG and its SpaC pilus adhesin using human fetal gut models.

\section{RESULTS}

\section{L. rhamnosus GG attenuates inflammatory cytokine production} in response to pathogenic bacteria and proinflammatory signals in the human fetal gut and IECs

To assess the antiinflammatory potential of L. rhamnosus GG in the fetal human gut, we examined its effect on Salmonella enterica serovar Typhimurium SL1344 (S. Typhimurium)induced tumor necrosis factor (TNF)- $\alpha$ mRNA expression in human fetal ileal organ culture. As shown in Figure 1a, TNF- $\alpha$ mRNA expression was increased 4.4 -fold $(P=0.049)$ compared to baseline after exposure to viable $S$. Typhimurium. On the other hand, live L. rhamnosus GG did not result in a significant increase in TNF- $\alpha$ mRNA levels (Figure 1a). However, when human fetal ileal organ culture was exposed to both $S$. Typhimurium and $L$. rhamnosus GG, TNF- $\alpha$ mRNA expression was reduced to -1.4 -fold compared to baseline $(P$ $=0.026)$. Similar results were obtained when $S$. Typhimuriuminduced IL-8 responses were measured on protein level in T84 cells (Figure 2a) suggesting that IECs are the target for potential probiotic action by L. rhamnosus GG.

To exclude the possibility that the observed reduction in inflammatory responsiveness might be the result of L. rhamnosus GG directly inhibiting $S$. Typhimurium, an experiment using lipopolysaccharide (LPS), a cell wall component of Gram-negative bacteria known to induce an inflammatory response through TLR4 signaling, was conducted (Figure 1b). After LPS stimulation, TNF- $\alpha$ mRNA expression in the fetal gut was increased 11.9-fold compared to baseline $(P=0.003)$.
Exposure to L. rhamnosus GG reduced this inflammatory response to 3.1 -fold $(P=0.009)$. In the fetal IEC line $\mathrm{H} 4$, exposure to live L. rhamnosus GG was observed to attenuate IL- $1 \beta$ and TNF- $\alpha$-induced IL- 6 secretion on protein level as assessed by ELISA (Figure $2 \mathrm{~b}, \mathrm{c}$ ) suggesting that fetal IECs are susceptible to immunomodulation by L. rhamnosus GG.

\section{L. rhamnosus GG SpaC pilus adhesin binds to IECs in the fetal human gut and modulates inflammatory cytokine responses}

To determine whether the adhesive SpaC pilin subunit of the L. rhamnosus GG SpaCBA pilus can bind to the human fetal intestinal epithelium, we used recombinant $\mathrm{SpaC}$ protein and assessed its adhesion capacity using both human fetal ileal organ culture and cell culture models. In the fetal ileum, binding of recombinant SpaC to the epithelial lining was detected by immunohistochemistry (Figure 3a,b). In a similar fashion, SpaC was observed to bind to the immature human intestinal epithelial cell line $\mathrm{H} 4$ by immunofluorescence (Figure $3 \mathrm{c}, \mathrm{d}$ ).

To test whether the binding of SpaC to IECs modulates inflammatory responses in fetal human IECs, $\mathrm{H} 4$ cells were incubated for $60 \mathrm{~min}$ with increasing concentrations of recombinant SpaC before stimulation with IL-1 $\beta$, a proinflammatory cytokine that shares the same intracellular signaling pathway with TLRs. A modest, dose-dependent reduction in inflammatory responses as assessed by IL- 6 protein secretion was observed after exposure to SpaC (Figure 4a). Interestingly, however, when $\mathrm{H} 4$ cells were stimulated with TNF- $\alpha$, a potent endogenous proinflammatory mediator using a distinct receptor and intracellular pathway, exposure to SpaC lead to a modest but statistically significant increase in IL-6 protein secretion (Figure 4b).

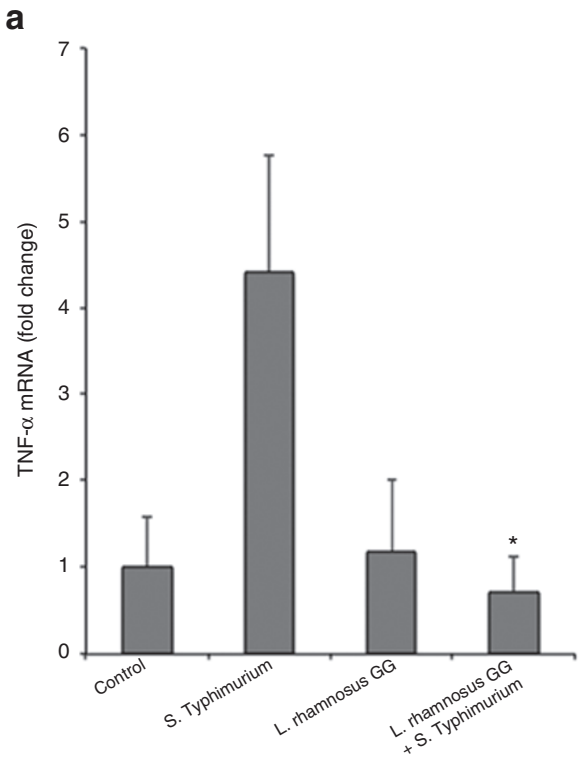

b

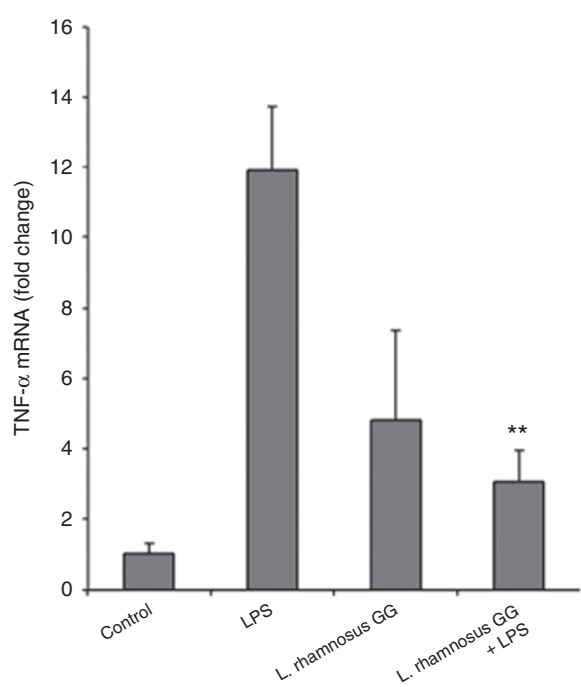

Figure 1. Live Lactobacillus rhamnosus GG attenuates inflammatory responses in the fetal gut. (a) Salmonella Typhimurium induces expression of TNF- $\alpha$ mRNA in human fetal intestinal culture which is significantly attenuated by live L. rhamnosus GG. (b) Lipopolysaccharide (LPS), a proinflammatory cell wall component of Gram negative organisms, also induces a strong TNF- $\alpha$ mRNA in response in the human fetal gut and this reaction is significantly decreased by live L. rhamnosus GG. $\left({ }^{*} P<0.05\right.$, ${ }^{* *} P<0.01$.) 


\section{L. rhamnosus GG SpaC pilus adhesin modulates immune gene expression in the human fetal gut}

To obtain a broader view of the influence of the SpaC pilus adhesin on innate immune responses, we evaluated how recombinant $\mathrm{SpaC}$ modulates immune gene expression in the human fetal gut. The data from these mRNA expression studies are summarized in Table 1 . After the 4-h exposure to recombinant $\mathrm{SpaC}$, we observed a range of variation in TLRrelated mRNA levels. In particular, the expression of mRNA for TLR3, TLR4 and the intracellular adapter molecule TIRAP was significantly downregulated. In contrast, no effect was seen in the mRNA expression for the antiinflammatory mediators IL-10 or TOLLIP. The expression of the IL-1 receptor antagonist IL1RN was significantly increased.

\section{DISCUSSION}

Our results indicate that the human fetal gut is responsive to microbes and microbial components and that these reactions are microbe-specific. A brisk inflammatory response was elicited in the human fetal gut towards $S$. Typhimurium, a pathogenic organism, whereas little or no increase in TNF- $\alpha$ mRNA expression was observed in response to the probiotic L. rhamnosus GG in this study. Furthermore, L. rhamnosus GG attenuated the inflammatory reaction launched against $S$. Typhimurium. These responses were consistently observed in fetal intestinal tissue obtained as early as 14-21 wk of gestation. It appears that IECs are the primary target for immunomodulation by L. rhamnosus GG, since exposure to live L. rhamnosus GG significantly reduced inflammatory chemokine production in response to indigenous proinflammatory stimuli in the fetal IEC H4 cell line.

The precise mechanisms by which probiotics exert their effects in the human gut are poorly understood despite a growing body of data from experimental studies. It has recently been discovered through genome sequencing and detailed studies of putative adhesins that the probiotic L. rhamnosus GG uses pili for adhesion and that adhesin SpaC, which is located on the pilus tip as well as decorates the shaft (13), is the major binding factor to human intestinal mucus and collagen as well as the adult human colonic intestinal epithelial a

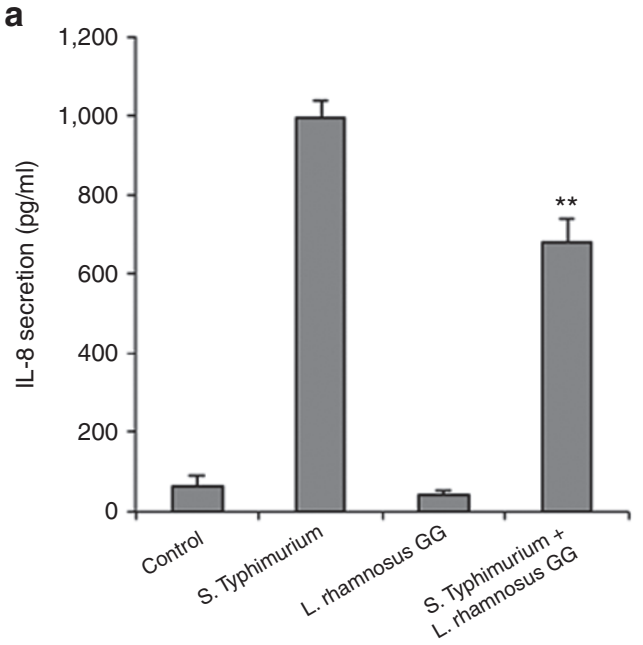

b

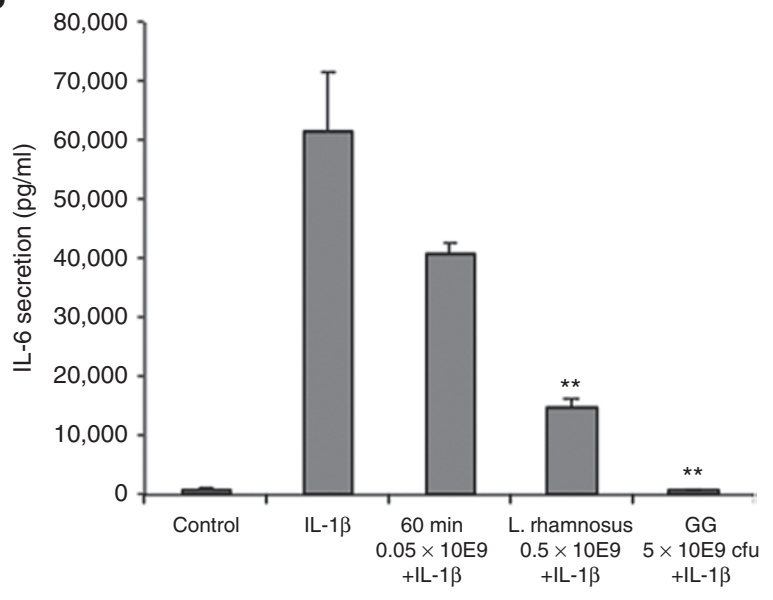

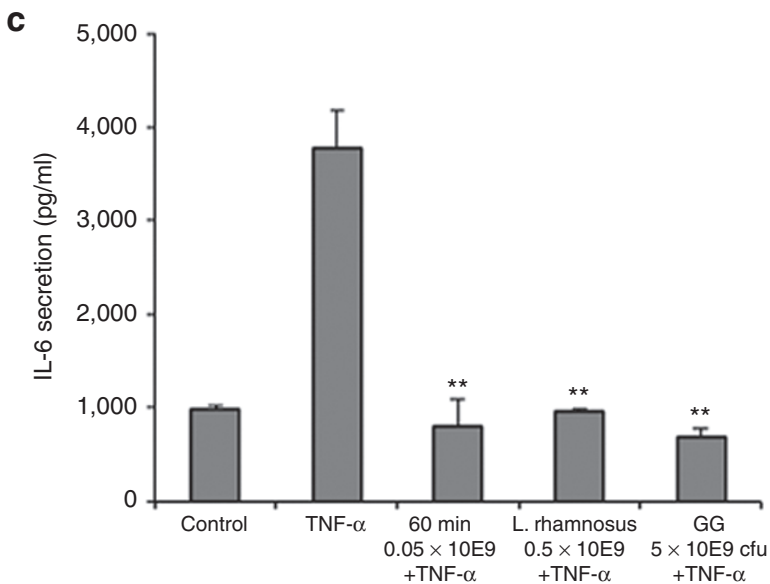

Figure 2. Live Lactobacillus rhamnosus GG attenuates inflammatory responses in IECs. Salmonella Typhimurium induces IL-8 secretion in the human adult intestinal epithelial cell line T84. (a) This response in significantly reduced by exposure to live L. rhamnosus GG. In a similar fashion, exposure to live L. rhamnosus GG significantly attenuates proinflammatory IL- 6 secretion elicited in response to the indigenous proinflammatory mediators (b) IL-1 $\beta$ or (c) TNF- $\alpha$ in the fetal intestinal epithelial cell line H4. ${ }^{* * P}<0.01$.) 
a

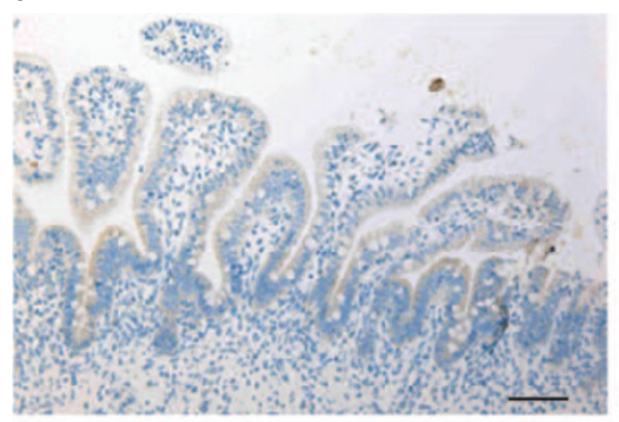

c

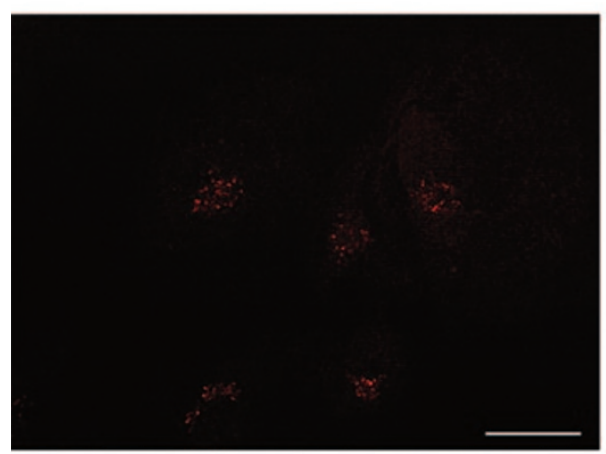

b

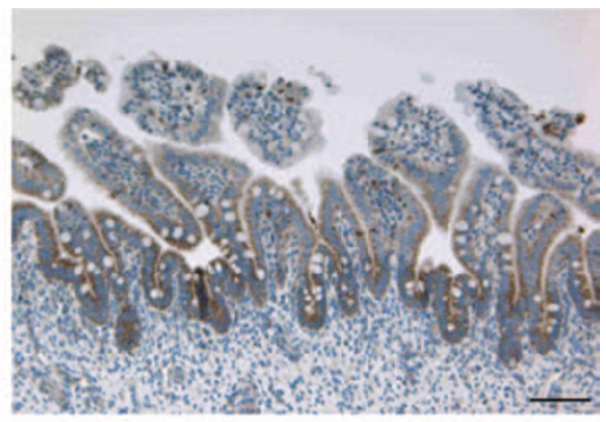

d

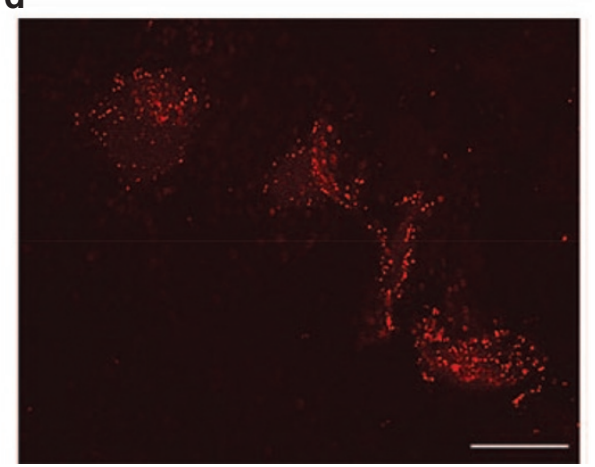

Figure 3. Recombinant Lactobacillus rhamnosus $\mathrm{GG}$ pilus adhesin SpaC binds to human fetal gut. After a 60 -min incubation with $10 \mu \mathrm{g} / \mathrm{ml}$ recombinant L. rhamnosus GG pilus adhesin SpaC, binding to the epithelium is observed in (b) human fetal intestinal organ culture as compared to (a) untreated control by immunohistochemistry (magnification 20x, the scale bars represent $50 \mathrm{~nm}$ ). In a similar fashion, SpaC binding is detected in the human fetal intestinal epithelial cell line $\mathrm{H} 4$ after exposure to (d) SpaC as compared to (c) untreated control by immunofluorescence (magnification 100x, the scale bars represent $15 \mathrm{~nm}$ ).

a

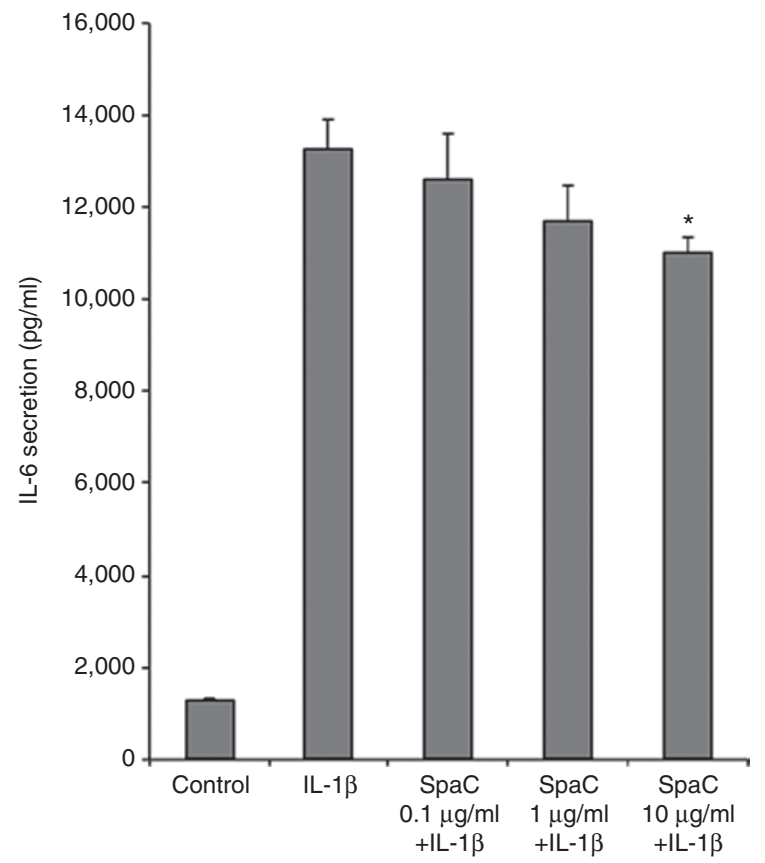

b

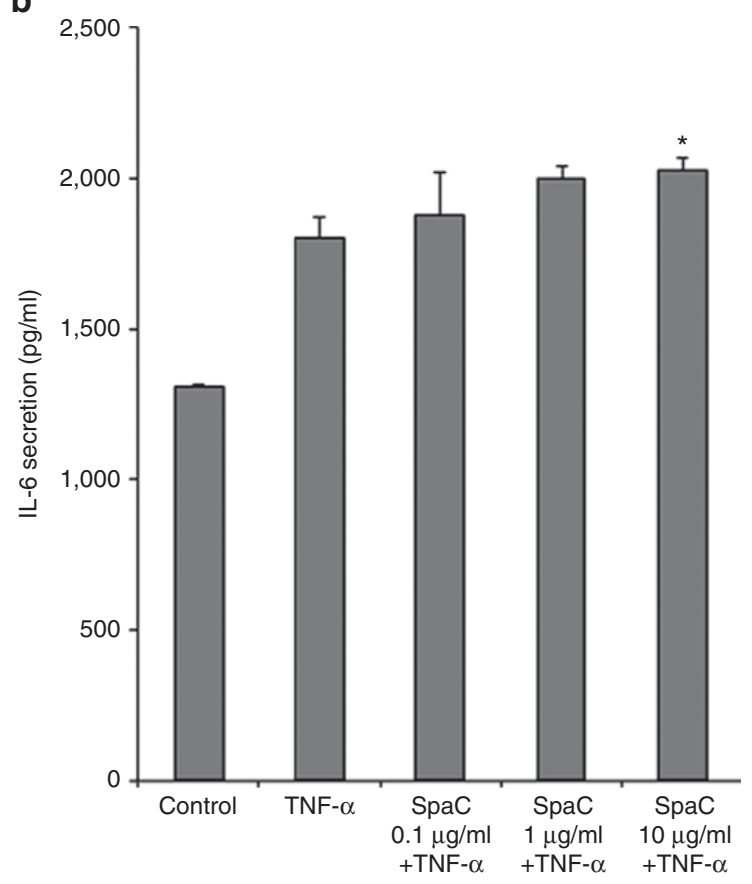

Figure 4. Exposure to recombinant Lactobacillus rhamnosus GG pilus adhesin SpaC attenuates inflammatory responses in the human fetal intestine. (a) A modest dose-dependent reduction in IL-1 $\beta$-induced IL-6 production is observed in the human fetal intestinal epithelial cell line $\mathrm{H} 4$ after a 60 -min exposure to SpaC. (b) In contrast, TNF- $\alpha$-induced IL- 6 secretion is modestly increased by exposure to SpaC. $\left({ }^{*} P<0.05\right.$.) 
Table 1. Effect of exposure to $10 \mu \mathrm{g} / \mathrm{ml}$ recombinant Lactobacillus rhamnosus GG SpaC pilus adhesin on immune gene expression in the fetal gut

\begin{tabular}{lrrl}
\hline Gene & Fold change & \multicolumn{1}{c}{$95 \% \mathrm{Cl}$} & \multicolumn{1}{c}{$P$} \\
\hline IL10 & 1.94 & $0.00001-4.24$ & \multicolumn{1}{c}{0.42} \\
ILIR1 & 1.32 & $0.69-1.95$ & 0.28 \\
ILIRAP & 0.96 & $0.69-1.22$ & 0.72 \\
ILIRN & 8.24 & $3.05-13.43$ & 0.028 \\
TLR1 & 0.40 & $0.11-0.69$ & 0.097 \\
TLR2 & 0.90 & $0.48-1.33$ & 0.77 \\
TLR3 & 0.28 & $0.09-0.48$ & 0.016 \\
TLR4 & 0.23 & $0.12-0.34$ & 0.026 \\
TLR5 & 1.27 & $0.00001-2.74$ & 0.79 \\
TLR6 & 0.92 & $0.18-1.66$ & 0.64 \\
TLR7 & 0.39 & $0.00001-0.90$ & 0.17 \\
TLR9 & 0.41 & $0.06-0.76$ & 0.15 \\
MD-2 & 0.54 & $0.00001-1.09$ & 0.38 \\
TIRAP & 0.46 & $0.39-0.52$ & 0.00032 \\
TOLLIP & 3.68 & $0.00001-2.85$ & 0.65 \\
\hline
\end{tabular}

cell line Caco-2 (10-12,14). In this study, we demonstrated that $\mathrm{SpaC}$ also binds to the fetal human gut and specifically to the fetal human small intestinal cell line $\mathrm{H} 4$.

In addition to adhesion, SpaC appears to modulate IEC function. A mutant of L. rhamnosus GG devoid of SpaC has previously been reported to induce increased mRNA expression for the proinflammatory cytokines IL- 8 and TNF- $\alpha$ in Caco- 2 cells while wild-type $L$. rhamnosus $\mathrm{GG}$ or $\mathrm{SpaC}$ alone had little impact on cytokine production (14). Our data indicate that $\mathrm{SpaC}$ exerts immunomodulatory effects also in the fetal gut. In line with the dose-dependent attenuation of IL-1 $\beta$ induced IL-6 secretion observed in H4 cells after exposure to live $L$. rhamnosus $\mathrm{GG}$, exposure to $\mathrm{SpaC}$ alone resulted in a more modest but dose-dependent reduction in IL-6 secretion in response to IL-1 $\beta$. Surprisingly, however, TNF- $\alpha$-induced IL-6 secretion was modestly increased in a dose-dependent fashion in $\mathrm{H} 4$ cells exposed to $\mathrm{SpaC}$, which is in stark contrast to the observed brisk attenuation of TNF- $\alpha$-induced IL-6 secretion after exposure to live L. rhamnosus GG. Taken together, we interpret these data to suggest that SpaC may play a role in mediating the antiinflammatory effects of live $L$. rhamnosus GG on the TLR/IL-1R signaling pathway in IECs. The models used in the present series of experiments do not allow assessing the role of gut immune cells or the immunologically active intestinal crypt cells in the immunomodulatory effects of $L$. rhamnosus GG. The mechanisms of attenuated TNF- $\alpha$-induced inflammatory responses in IECs also remain to be elucidated.

The observed L. rhamnosus GG-induced attenuation of fetal gut inflammatory responses against $S$. Typhimurium or LPS may in part be mediated by modulation of immune gene expression by the $\mathrm{SpaC}$ pilus adhesin. Expression of
mRNA for TLR4, a receptor that specifically binds LPS and therefore initiates responses to many Gram-negative bacteria, was significantly reduced by exposure to $\mathrm{SpaC}$ in the fetal gut. Furthermore, SpaC also downregulated the expression of IL-1 receptor antagonist (IL1RN) and TIRAP, an adaptor molecule involved in the common TLR and IL-1 receptor signaling pathway. We have reported similar effects on TLR and TIRAP mRNA expression in the placenta and the fetal gut after maternal consumption of a probiotic combination including L. rhamnosus GG in a clinical trial (5). We speculate that the observed reduction in IL- $1 \beta$-induced IL- 6 secretion in H4 cells after exposure to live L. rhamnosus GG or SpaC may result from modulation of the common TLR and IL-1 receptor signaling pathway. Probiotic lactobacilli have been observed to modulate TLR gene expression and induce the expression of the TLR/IL1R interacting protein TOLLIP and immunoregulatory cytokines in human cord blood-derived dendritic cells (16). We have previously reported that secreted products from probiotic lactobacilli and bifidobacteria downregulate TLR2 and TLR4 expression and attenuate inflammatory responses through a TOLLIP-dependent mechanism in the immature human gut (17). In this study, however, the expression of TOLLIP and the immunoregulatory cytokine IL-10 were unaltered by exposure to $\mathrm{SpaC}$ suggesting that these mechanisms were not the primary target of SpaC.

Previously, intrauterine microbial contact has mostly been investigated in the context of invading pathogens. It is well established that colonization of the amniotic membranes by microbes such as Ureaplasma urealyticum or Mycoplasma hominis increases the risk of premature rupture of membranes and preterm birth (18). Bacterial chorionamnionitis, an important cause of preterm birth, is also associated with significant morbidities including fetal and maternal infection as well as bronchopulmonary dysplasia and cerebral palsy in the infant $(1,2)$. All of this notwithstanding, live bacteria have also been detected in the placenta at $23-28$ wk of gestation after caesarean section delivery with no signs of infection or labor (19). In healthy term pregnancies, bacteria have frequently been detected in the chorionamnion, amniotic fluid, and placenta after caesarean section without rupture of membranes (3-5). This challenges the often-repeated notion of sterile fetal life. The origin and significance of microbes in the fetoplacental unit remains unknown but there are data to suggest active transfer of bacteria from the maternal gut. The composition of maternal gut microbiota has been observed to change dramatically during pregnancy (20) and it has been hypothesized that these changes may be causally related to infant gut immune function (21). An increase in intestinal bacterial translocation has been observed in experimental animals during pregnancy (22) and labeled Enterococcus faecium introduced to the maternal gut has been detected in the amniotic fluid in an experimental mouse model (23).

The significance of prenatal microbial contact to human health was initially recognized in epidemiological studies linking maternal contact with farm animals, and thus presumably 
increased maternal microbial exposure during pregnancy, with altered TLR-related gene expression and decreased incidence of atopic disease in the infant (24). Experiments conducted using a mouse model suggest that maternal contact with a bacterium isolated from cow-sheds, Acinetobacter lwoffii F78, modulates placenta immune function and decreases the risk of developing asthma in the offspring (25). In addition to these indirect effects of maternal microbial contact, direct exposure to nonpathogenic bacteria has been reported to modulate fetal and neonatal immunophysiology. In a pioneer study by Fichorova et al., an association between bacteria in the human placenta and systemic immune responses in extremely preterm neonates was reported (7). Interestingly, the presence of lactobacilli in the placenta was observed to be associated with a reduced neonatal inflammatory response whereas pathogenic bacteria increased markers of systemic inflammation in the study (7). In addition to systemic immune responses, prenatal microbial contact appears to modulate human fetal intestinal immunophysiology, since we have recently reported that the presence of DNA from nonpathogenic bacteria in the placenta is associated with alterations in TLR-related innate immune gene expression in the human fetal gut (5). These data are consistent with our in vitro findings showing reduced inflammatory responses and altered TLR-related gene expression after exposure to L. rhamnosus GG or SpaC in fetal human gut models. Our present data for the first time provide experimental and mechanistic insight into host-microbe interactions in the human fetus.

Data from clinical trials suggest that prenatal maternal probiotic supplementation may enhance the efficacy of probiotic interventions aiming to reduce the risk of atopic dermatitis in the infant $(8,9)$. It is intriguing to speculate that indirect modulation of fetal immune function may be one of the mechanisms by which prenatal probiotic intervention exerts its effects. Future research efforts should be aimed to investigate the microbiologic and immunologic effects in the fetoplacental unit during maternal probiotic supplementation in both experimental and clinical settings. Given the safety concerns raised regarding the use of viable probiotics, particularly in preterm neonates, the use of specific components of probiotic bacteria such as $\mathrm{SpaC}$ with specific effects may provide a novel means of improving infant health in the future.

\section{METHODS}

\section{Reagents}

Media (DMEM/F12, CMRL, and Opti-MEM I) and other reagents for tissue and cell culture were obtained from Gibco-Invitrogen (Carlsbad, $\mathrm{CA})$. Hydrocortisone hemisuccinate, tricine, $\beta$-retinyl acetate, collagenase type IV, protease inhibitor cocktail, phosphatase inhibitor cocktail I and II were obtained from Sigma-Aldrich (St Louis, MO). Fetal bovine serum (FBS) was obtained from Atlanta Biologicals (Lawrenceville, GA). Recombinant human insulin (Novolin R) was obtained from Novo Nordisk A/S (Bagsvaerd, Denmark). Extracellular matrix ECL was obtained from Upstate Biotechnology (Lake Placid, NY). The cytokines IL- $1 \beta$ and TNF- $\alpha$ and all ELISA reagents were obtained from R\&D Systems (Minneapolis, MN). Cy 3-conjugated $\mathrm{F}(\mathrm{ab})_{2}$ fragment goat antirabbit IgG was obtained from Jackson ImmunoResearch (West Grove, PA). The Vectastain universal Elite $\mathrm{ABC}$ kit and 3,3'-diaminobenzidine were obtained from Vector
Laboratories (Burlingame, CA). Permount was obtained from Fisher Chemicals (Fair Lawn, NJ). Human recombinant epidermal growth factor (EGF), Trizol, and SuperScript III Platinum SYBR Green OneStep qRT-PCR kits were obtained from Invitrogen (Carlsbad, CA). All other reagents were of analytical or molecular biology grade and obtained from Sigma-Aldrich.

\section{Human Fetal Intestinal Models}

The study was conducted according to the National Institutes of Health (NIH, Bethesda, MD) guidelines and Partners Human Research Committee (Institutional Review Board for the Massachusetts General Hospital, Boston, MA) approval (IRB\# 1999p003833). Fetal ileal organ culture was prepared from intestinal tissue obtained from therapeutic abortions using a protocol modified from that described previously (26). Briefly, fetal small intestine was stripped of its mesentery and cut into explants which were cultured in a Falcon organ culture dish at $37^{\circ} \mathrm{C}$ with $95 \% \mathrm{O}_{2}$ and $5 \% \mathrm{CO}_{2}$ atmosphere saturated with water vapor. In experiments using live bacteria, $20-30 \mathrm{~mm}$ pieces of small intestine were rinsed with antibiotic free medium after which the lumen was injected with antibiotic-free medium supplemented with live bacteria (please see below). The explant ends were closed with sterile sutures and the explants were then suspended in medium containing antibiotics. The purpose of these measures was to ensure that only the luminal side of the intestinal explant comes into contact with live bacteria. The culture medium consisted of CMRL 1066 supplemented with $5 \% \mathrm{FBS}, 5 \mathrm{~g} / \mathrm{l}$ glucose, $20 \mathrm{mmol} / \mathrm{l}$ tricine buffer ( $\mathrm{pH} 7.4) 0.5 \mu \mathrm{g} / \mathrm{l}$ hydrocortisone hemisuccinate, $1 \mathrm{mg} / \mathrm{l} \mathrm{b}$-retinyl acetate, $50 \mathrm{U} / \mathrm{ml}$ penicillin and $50 \mu \mathrm{g} / \mathrm{ml}$ streptomycin. The tissues were allowed to equilibrate for $30 \mathrm{~min}$ before beginning the experiments. The tissue explants have been shown to remain viable up to $48 \mathrm{~h}$ (26). Tissue viability was also assessed indirectly by mRNA recovery at the time of sample collection (data not shown).

The nontransformed primary human fetal intestinal epithelial cell line $\mathrm{H} 4$ was used in subsequent studies. The cell line has previously been characterized in detail (27). The cells are morphologically immature and do not polarize or form tight junctions. The H4 culture medium consisted of DMEM supplemented with 5\% heat-inactivated fetal bovine serum, $5 \%$ heat-inactivated neonatal bovine serum, $1 \%$ glutamine, $1 \%$ sodium pyruvate, $1 \%$ nonessential amino acids, $1 \%$ HEPES, $0.2 \mathrm{U} / \mathrm{ml}$ insulin, $50 \mathrm{U} / \mathrm{ml}$ penicillin, and $50 \mu \mathrm{g} / \mathrm{ml}$ streptomycin. In addition, the adult colon cancer cell line T84, which polarize and form tight junctions, was used for experiments with live bacteria. The T84 cell culture medium consisted of DMEM supplemented with $5 \%$ heat-inactivated fetal bovine serum, $1 \%$ glutamine, $2.5 \%$ HEPES, $50 \mathrm{U} / \mathrm{ml}$ penicillin, and $50 \mu \mathrm{g} / \mathrm{ml}$ streptomycin. The cells were incubated in culture dishes at 37 ${ }^{\circ} \mathrm{C}, 95 \% \mathrm{O}_{2}$ and $5 \% \mathrm{CO}_{2}$ atmosphere saturated with water vapor.

\section{Inflammatory Responses in the Human Fetal Intestinal Culture Model}

Human fetal intestinal organ culture was performed as described above. The medium was supplemented with either $5 \times 10^{9} \mathrm{cfu} / \mathrm{ml}$ live L. rhamnosus GG or S. Typhimurium or with both strains in combination. In an independent series of experiments, the impact of live $L$. rhamnosus GG on inflammatory responses induced by $50 \mathrm{ng} / \mathrm{ml} \mathrm{LPS}$ was assessed and compared to unsupplemented medium. After $4 \mathrm{~h}$, the explants were rapidly frozen in liquid nitrogen and stored in -80 ${ }^{\circ} \mathrm{C}$. Total cellular RNA was extracted from homogenized tissue by the Trizol-chloroform extraction method as described previously (26). The levels of TNF- $\alpha$ and IL- 8 mRNA was measured in duplicate for each sample by quantitative real-time polymerase chain reaction (qRT-PCR) using the SuperScript III Platinum SYBR Green One-Step qRT-PCR kit with MJ Opticon 2 DNA engine (MJ Research, Waltham, MA) according to manufacturer's instructions. OpticonMONITOR analysis software version 2.01 (MJ Research) was implemented to normalize the levels of TNF- $\alpha$ mRNA to the standard GAPDH level for each sample.

\section{Inflammatory Responses in Cell Culture Models}

T84 cells were grown to $90 \%$ confluence and incubated in antibiotic-free medium for $60 \mathrm{~min}$ before exposure to $5 \times 10^{9} \mathrm{cfu} / \mathrm{ml}$ live L. rhamnosus GG for $60 \mathrm{~min}$. The cells were subsequently stimulated with $5 \times 10^{9} \mathrm{cfu} /$ 
$\mathrm{ml}$ live $S$. Typhimurium for $60 \mathrm{~min}$. H4 cells were grown to $70 \%$ confluence before exposure to indicated concentrations of live L. rhamnosus GG or recombinant SpaC purified as described previously $(10,12)$ for $60 \mathrm{~min}$. Subsequently, the cells were stimulated with either $1 \mathrm{ng} / \mathrm{ml}$ IL- $1 \beta$ or $10 \mathrm{ng} / \mathrm{ml}$ TNF- $\alpha$ as indicated. In all instances, unstimulated cells were used as control. All experiments were performed in triplicate. After $18 \mathrm{~h}$, the culture medium was collected, centrifuged and stored at $-20^{\circ} \mathrm{C}$ for measurement of IL- 8 or IL- 6 by ELISA as described previously (28).

\section{SpaC Binding to Human Fetal Gut}

Human fetal intestinal organ culture was prepared as described above and the explants were cultured in medium containing $10 \mu \mathrm{g} /$ $\mathrm{ml}$ recombinant SpaC. Medium alone served as control. After $60 \mathrm{~min}$, the explants were rapidly frozen in liquid nitrogen and stored in -80 ${ }^{\circ} \mathrm{C}$. Immunohistochemical staining was performed as described previously (29). Briefly, series of $5 \mu \mathrm{m}$ thick sections were cut from frozen tissue samples embedded in optimum cutting temperature compound. Immunostaining was performed by incubation with polyclonal rabbit anti-SpaC antibody diluted 1:100 for $60 \mathrm{~min}$ followed by incubation with Vectastain universal Elite $\mathrm{ABC}$ kit with 3,3'-diaminobenzidine as chromogen for $60 \mathrm{~min}$ employing the protocol and reagents recommended by the manufacturer for frozen sections. Tissues were counterstained in Harris hematoxylin and mounted with Permount. All incubations were performed at room temperature. The specificity of the immunoreaction was monitored by replacing the primary antibody with nonimmune sera.

To assess $\mathrm{SpaC}$ binding to human fetal IECs, $\mathrm{H} 4$ cells were grown to $70 \%$ confluency on cover slips and exposed to $10 \mu \mathrm{g} / \mathrm{ml} \mathrm{recombinant}$ SpaC. Medium alone served as control. After $60 \mathrm{~min}$, the cells were washed three times using phosphate-buffered saline supplemented with $\mathrm{Mg}$ and $\mathrm{Ca}$ and then fixed in $4 \%$ paraformaldehyde for $30 \mathrm{~min}$ on ice. Once permeabilized with methanol ( $10 \mathrm{~min}$ on ice) and blocked with $10 \%$ goat serum in Tris-buffered saline containing $0.25 \%$ bovine serum albumin, the cells were incubated overnight with a rabbit antiSpaC polyclonal antibody $(10,12)(1: 2,000)$ in Tris-buffered saline with $0.25 \%$ bovine serum albumin. After washing, the cells were incubated with a Cy 3 -conjugated goat antirabbit $\operatorname{IgG}\left(\mathrm{F}\left(\mathrm{ab}^{\prime}\right)_{2}(1: 2,000)\right.$ in Tris-buffered saline with $0.25 \%$ bovine serum albumin and examined using a Zeiss Axiophot photomicroscope (Germany) and a digital image was obtained. Purified rabbit IgG was used as a control.

\section{Effect of SpaC on Innate Immune Gene Expression in the Human Fetal Gut}

Human fetal intestinal organ culture was prepared as described above and the explants were cultured in medium containing $10 \mu \mathrm{g} / \mathrm{ml} \mathrm{recom-}$ binant SpaC. Medium alone served as control. After $4 \mathrm{~h}$, the explants were rapidly frozen in liquid nitrogen and stored in $-80^{\circ} \mathrm{C}$. For TLRrelated gene expression analyses, total RNA was isolated from fetal ileal organ culture explants using the Trizol reagent (TriReagent, Molecular Research Center, Cincinnati, $\mathrm{OH}$ ) according to the manufacturer's instructions, followed by a purification step using Qiagen RNAeasy mini kit (Qiagen, Hilden, Germany) columns according to the manufacturer's instructions. Total RNA concentrations and A260/A280 and A260/A230 ratios were determined using a NanoDrop ND1000 spectrophotometer to ensure the integrity and purity of the mRNA.

Expression of genes encoding IL-1 receptor 1 (IL1R1), IL-1 receptor accessory protein (IL1RAP), IL-1 receptor antagonist (IL1RN), TLRs 1-7, TLR9, and the TLR4 coreceptor MD-2, the TLR adaptor protein TIRAP as well as the negative regulator TOLLIP and antiinflammatory cytokine IL-10 in fetal human ileum was assessed using $\mathrm{RT}^{2}$ Profiler PCR Array (PAHS-077, SA Biosciences, Qiagen, Hilden, Germany). For cDNA synthesis, $5 \mu \mathrm{g}$ total RNA was used with the RT2 First Strand Kit (SA Biosciences) as described by the manufacturer. The qPCR array analysis was carried out following the manufacturer's protocol with SYBR Green PCR (ROX) Master Mix (Applied Biosystems, Foster City, CA) on ABI 7300 Real Time PCR System.

\section{Statistical Analyses}

TNF- $\alpha$ mRNA expression data obtained by qRT-PCR are expressed as geometric means with SE after logarithmic transformation; comparisons between groups were performed using Student's two-tailed $t$-test after logarithmic transformation. Secretion of IL- 8 and IL-6 in cell culture experiments was expressed as means $\pm \mathrm{SE}$; comparisons between groups were performed using two-tailed Student's $t$-test. Differences in the expression of TLR-related genes were determined using the $2-\Delta \Delta \mathrm{Ct}$ method. $\Delta \mathrm{Ct}$ values were calculated by subtracting individual $\mathrm{Ct}$ values from the Ct values of the most stable housekeeping gene, which in turn was determined using the normfinder algorithm. The expressional pattern differences were generated using the expressional fold differences analyzed through the SA Biosciences web page. A $P$ value $<0.05$ was considered statistically significant.

\section{ACKNOWLEDGMENTS}

All authors were involved in designing the research, data analyses, and writing the report. All authors take responsibility for the final report.

\section{STATEMENT OF FINANCIAL SUPPORT}

The project was supported by the Academy of Finland and the Emil Aaltonen Foundation. The work carried out at the University of Helsinki was supported by an Academy of Finland general research grant (118165) and by the Academy of Finland-funded Center of Excellence in Microbial Food Safety (CoE-MiFoSa) research program (141140). The work conducted at Massachusetts General Hospital for Children was supported by program project grant from the National Institute of Diabetes and Digestive and Kidney Diseases (NIDDK, Bethesda, MD) (P01-DK033506-30-P.I. W.A.W.).

Disclosure: There is no conflict of interest to disclose.

\section{REFERENCES}

1. Rautava S, Luoto R, Salminen S, Isolauri E. Microbial contact during pregnancy, intestinal colonization and human disease. Nat Rev Gastroenterol Hepatol 2012;9:565-76.

2. Goldenberg RL, Culhane JF, Iams JD, Romero R. Epidemiology and causes of preterm birth. Lancet 2008;371:75-84.

3. Steel JH, Malatos S, Kennea N, et al. Bacteria and inflammatory cells in fetal membranes do not always cause preterm labor. Pediatr Res 2005;57: 404-11.

4. Satokari R, Grönroos T, Laitinen K, Salminen S, Isolauri E. Bifidobacterium and Lactobacillus DNA in the human placenta. Lett Appl Microbiol 2009;48:8-12.

5. Rautava S, Collado MC, Salminen S, Isolauri E. Probiotics modulate hostmicrobe interaction in the placenta and fetal gut: a randomized, doubleblind, placebo-controlled trial. Neonatology 2012;102:178-84.

6. Mshvildadze M, Neu J, Shuster J, Theriaque D, Li N, Mai V. Intestinal microbial ecology in premature infants assessed with non-culture-based techniques. J Pediatr 2010;156:20-5.

7. Fichorova RN, Onderdonk AB, Yamamoto $\mathrm{H}$, et al.; Extremely Low Gestation Age Newborns (ELGAN) Study Investigators. Maternal microbe-specific modulation of inflammatory response in extremely lowgestational-age newborns. MBio 2011;2:e0280-10.

8. Isolauri E, Rautava S, Salminen S. Probiotics in the development and treatment of allergic disease. Gastroenterol Clin North Am 2012;41:747-62.

9. Rautava S, Kainonen E, Salminen S, Isolauri E. Maternal probiotic supplementation during pregnancy and breast-feeding reduces the risk of eczema in the infant. J Allergy Clin Immunol 2012;130:1355-60.

10. von Ossowski I, Reunanen J, Satokari R, et al. Mucosal adhesion properties of the probiotic Lactobacillus rhamnosus GG SpaCBA and SpaFED pilin subunits. Appl Environ Microbiol 2010;76:2049-57.

11. Tripathi P, Beaussart A, Alsteens D, et al. Adhesion and nanomechanics of pili from the probiotic Lactobacillus rhamnosus GG. ACS Nano 2013;7:3685-97.

12. Kankainen M, Paulin L, Tynkkynen S, et al. Comparative genomic analysis of Lactobacillus rhamnosus GG reveals pili containing a human- mucus binding protein. Proc Natl Acad Sci USA 2009;106:17193-8.

13. Reunanen J, von Ossowski I, Hendrickx AP, Palva A, de Vos WM. Characterization of the SpaCBA pilus fibers in the probiotic Lactobacillus rhamnosus GG. Appl Environ Microbiol 2012;78:2337-44.

14. Lebeer S, Claes I, Tytgat HL, et al. Functional analysis of Lactobacillus rhamnosus GG pili in relation to adhesion and immunomodulatory interactions with intestinal epithelial cells. Appl Environ Microbiol 2012;78:185-93. 
15. von Ossowski I, Pietilä TE, Rintahaka J, et al. Using recombinant Lactococci as an approach to dissect the immunomodulating capacity of surface piliation in probiotic Lactobacillus rhamnosus GG. PLoS ONE 2013;8:e64416.

16. Bermudez-Brito M, Muñoz-Quezada S, Gomez-Llorente C, et al. Human intestinal dendritic cells decrease cytokine release against Salmonella infection in the presence of Lactobacillus paracasei upon TLR activation. PLOS ONE 2012;7:e43197.

17. Ganguli K, Meng D, Rautava S, Lu L, Walker WA, Nanthakumar N. Probiotics prevent necrotizing enterocolitis by modulating enterocyte genes that regulate innate immune-mediated inflammation. Am J Physiol Gastrointest Liver Physiol 2013;304:G132-41.

18. DiGiulio DB. Diversity of microbes in amniotic fluid. Semin Fetal Neonatal Med 2012;17:2-11.

19. Onderdonk AB, Delaney ML, DuBois AM, Allred EN, Leviton A. Detection of bacteria in placental tissues obtained from extremely low gestational age neonates. Am J Obstet Gynecol 2008;198:110 e1-7.

20. Koren O, Goodrich JK, Cullender TC, et al. Host remodeling of the gut microbiome and metabolic changes during pregnancy. Cell 2012;150: 470-80.

21. Konstantinov SR, van der Woude CJ, Peppelenbosch MP. Do pregnancyrelated changes in the microbiome stimulate innate immunity? Trends Mol Med 2013;19:454-9.
22. Perez PF, Doré J, Leclerc M, et al. Bacterial imprinting of the neonatal immune system: lessons from maternal cells? Pediatrics 2007;119:e724-32.

23. Jiménez E, Fernández L, Marín ML, et al. Isolation of commensal bacteria from umbilical cord blood of healthy neonates born by cesarean section. Curr Microbiol 2005;51:270-4.

24. Roduit C, Wohlgensinger J, Frei R, et al.; PASTURE Study Group. Prenatal animal contact and gene expression of innate immunity receptors at birth are associated with atopic dermatitis. J Allergy Clin Immunol 2011;127:179-85, 185.e1.

25. Conrad ML, Ferstl R, Teich R, et al. Maternal TLR signaling is required for prenatal asthma protection by the nonpathogenic microbe Acinetobacter lwoffii F78. J Exp Med 2009;206:2869-77.

26. Nanthakumar NN, Fusunyan RD, Sanderson I, Walker WA. Inflammation in the developing human intestine: A possible pathophysiologic contribution to necrotizing enterocolitis. Proc Natl Acad Sci USA 2000;97: 6043-8.

27. Sanderson IR, Ezzell RM, Kedinger M, et al. Human fetal enterocytes in vitro: modulation of the phenotype by extracellular matrix. Proc Natl Acad Sci USA 1996;93:7717-22.

28. Claud EC, Savidge T, Walker WA. Modulation of human intestinal epithelial cell IL-8 secretion by human milk factors. Pediatr Res 2003;53:419-25.

29. Rautava J, Jee KJ, Miettinen PJ, et al. ERBB receptors in developing, dysplastic and malignant oral epithelia. Oral Oncol 2008;44:227-35. 